\title{
Accuracy of Uric Acid and Cholesterol Levels Examination in Distinguishing Pleural Effusion Fluid Exudates and Transudates
}

\author{
Yusup Subagio Sutanto \\ Department of Pulmonology and Respiratory Medicine, Faculty of Medicine, \\ Universitas Sebelas Maret, Dr. Moewardi Hospital, Surakarta
}

\section{ABSTRACT}

Background: Light's criteria was reported $25 \%$ of misclassification transudates as exudates. This study aimed to analyse the accuracy of examining uric acid levels and pleural fluid uric acid levels, and pleural fluid cholesterol and cholesterol ratios to distinguish the exudates and transudates in pleural effusions.

Subjects and Method: This was a crosssectional study design conducted at Dr. Moewardi Hospital, Surakarta, Central Java, from July to August 2019. The study subjects were 30 pleural effusion patients treated in the pulmonology ward. The dependent variables were pleural fluid exudates and transudates. The independent variables were (1) Uric acid levels in pleural fluid; (2) The ratio of uric acid levels between pleural fluid and serum; (3) Pleural fluid cholesterol levels; and (4) The ratio of cholesterol levels between pleural fluid and serum. The study instruments were Light's criteria and laboratory examination. The diagnosis's accuracy was analysed using sensitivity, specificity, and the area under the ROC (AUC) curve.

Results: Pleural fluid cholesterol showed sensitivity and specificity of $86 \%$ and $83 \%$, with a cut-off of 32.00 for transudate results. AUC value $=0.82$ with $p=0.012$. Serum cholesterol showed sensitivity and specificity of $71 \%$ and $61 \%$, with a cut-off of 175.50 for transudate results. AUC value $=0.67$ with $\mathrm{p}=0.194$. Pleural fluid uric acid levels showed a sensitivity and specificity of $86 \%$ and $87 \%$, with a cut-off of 7.25 for transudate results. AUC value $=0.83$ with $\mathrm{p}=0.009$. Examination of serum uric acid levels showed a sensitivity and specificity of $86 \%$ and $70 \%$, with a cut-off of 7.10 for transudate results. $\mathrm{AUC}$ value $=0.65$ with $\mathrm{p}=0.249$. Conclusion: Examination of uric acid and pleural fluid cholesterol levels can be used in routine pleural effusion examinations to distinguish exudates and transudates.

Keywords: accuracy, uric acid, exudates, cholesterol, transudates

\section{Correspondence:}

Yusup Subagio Sutanto. Department of Pulmonology and Respiratory Medicine, Faculty of Medicine Universitas Sebelas Maret, Dr. Moewardi Hospital, Surakarta. Jl. Kolonel Sutarto 132, Jebres, Surakarta 57126, Central Java. Email: dr_yusupsubagio@yahoo.com. Mobile: +62811284165 .

Cite this as:

Sutanto YS (2021). Accuracy of Checking Uric Acid and Cholesterol Levels in Distinguishing Pleural Effusion Fluid Exudates and Transudates. Indones J Med. 06(02): 159-167. https://doi.org/10.26911/theijmed.2021.06.02.05.

\section{BACKGROUND}

Pleural effusion is the accumulation of excess fluid in the pleural cavity, a common complication of both local and systemic disease processes. There are two types of effusions, namely exudative and transudative.
The pleural effusion diagnosis is made by identifying the fluid from the pleural puncture to determine the transudate or exudate. Research showed that there were limitations to Light's criteria, namely the mis- 
classification of transudates as exudates by 25\% (Kumar et al., 2020).

Light's criterion is frequently used to determine exudate and transudate effusions. Exudate pleural effusions meet at least one of Light's three criteria, whereas transudate effusions do not meet one criteria (Maskell and Butland, 2003). Light's criteria include (1) Pleural fluid protein to serum protein ratio $\geq 0.5$; (2) Ratio of pleural fluid lactate dehydrogenase (LDH) to serum $\mathrm{LDH} \geq 0.6$; and (3) pleural fluid $\mathrm{LDH}$ fluid $\geq 0.67$ from the normal upper limit of serum (Porcel, 2013; Oliveira and Burini, 2012).

One study reported that Light's criteria had high sensitivity (98\%) and low specificity (77\%). Several recent studies evaluated alternative criteria for differentiating transudates from exudates by examining pleural fluid cholesterol levels, serumpleural fluid albumin gradient, pleural fluid to serum cholesterol ratio, and bilirubin levels. Pleural fluid cholesterol can be used to classify exudates and transudates with less case misclassification than other Light parameters (Uzun, 2000).

Heffner et al. (2002) had identified an exudative type pleural effusion with at least one of the following conditions: (1) Pleural fluid protein $>2.9 \mathrm{mg} / \mathrm{dL}$; (2) Pleural fluid cholesterol $>45 \mathrm{mg} / \mathrm{dL}(1.16 \mathrm{mmol} / \mathrm{L})$; and (3) pleural fluid $\mathrm{LDH}>2 / 3$ of the upper limit of serum.

Elevated serum uric acid levels have also been observed in hypoxic states, such as obstructive pulmonary disease, cyanotic heart disease, and acute heart failure. Depletion of cellular $\mathrm{O}_{2}$, as in the case of hypoxia or hypoxic ischemia, results in the degradation of adenosine triphosphate (ATP) into hypoxanthine, xanthine, and uric acid catabolites (Uzun, 2000).

Uric acid is soluble in liquid media. An increase in uric acid levels in blood plas- ma is a predisposing factor for the accumulation of uric crystals in tissues and body fluids (Bansal et al., 2010; Yalcin et al., 2013).

There were several hypotheses regarding cholesterol in the pleural cavity to be used as a parameter of exudate. Acute inflammation of the pleura makes cholesterol enter and trapped in the pleural cavity, then changes its lipoprotein binding characteristics (Antony, 2003; Peng, 2004).

This study aimed to analyze the examination of pleural fluid uric acid levels and the ratio of pleural fluid uric acid levels to serum as well as pleural fluid cholesterol levels and pleural fluid to serum cholesterol levels to differentiate exudates and transudates in pleural effusions. This study's results were expected to be useful in providing an alternative examination of pleural fluid uric acid and cholesterol levels to be included in routine examinations for pleural effusion analysis.

\section{SUBJECTS AND METHOD}

\section{Study Design}

This diagnostic test research was conducted using a cross-sectional study design, from July to August 2019, in the outpatient and inpatient room for lung disease at the Dr. Moewardi Regional General Hospital, Surakarta, Central Java.

\section{Population dan Sample}

The study's source population (reachable population) of the study was patients who were admitted to clinic and the lung disease treatment room at Dr. Moewardi Hospital. Samples were selected using a consecutive sampling technique in pleural effusion patients. Pleural effusion was based on a diagnostic pleural puncture. The sample size was 30 patients with positive pleural effusions.

The inclusion criteria were the patient's age at the time of sampling $\geq 18$ 
years, the patient had been diagnosed as a pleural effusion patient, and was willing to participate in the study. Exclusion criteria were pleural effusion patients who refused to participate in the study. Subjects who met the inclusion criteria were then given education and data recording, including identity, history, physical examination, and laboratory tests, namely pleural fluid uric acid levels, pleural fluid uric acid levels to serum, pleural fluid cholesterol levels, pleural fluid to serum cholesterol levels, and pleural fluid analysis.

\section{Study Variables}

The dependent variables were pleural fluid exudates and transudates. The independent variables were pleural fluid uric acid levels, pleural fluid uric acid levels to serum, pleural fluid cholesterol levels, and pleural fluid to serum cholesterol levels.

\section{Operational Definition of Variables} Pleural fluid transudate was a transudative pleural effusion that occurs when systemic factors influence changes in pleural fluid formation or absorption. Transudates resulted from imbalances in hydrostatic and oncotic forces caused by some known clinical conditions such as heart failure and cirrhosis. The pleural surface was not involved in the primary pathological process.

The diagnosis of transudative effusion was confirmed by examining the characteristics of the pleural fluid. The transudate has the following three characteristics: (1) The ratio of pleural fluid to serum protein $<0.5$; (2) Ratio of pleural fluid LDH to serum LDH <0.6; and (3) Pleural fluid LDH value $<0.67$ from the upper limit of normal serum LDH.

The pleural fluid exudate was the addition of pleural fluid, which was influenced by local factors in the pleural cavity that affected pleural fluid accumulation chang- es. Exudates can occur in pneumonia, malignancy, thromboembolism, and others.

The diagnosis of pleural effusion exudative was made if it met one of the following three criteria: (1) The ratio of pleural fluid protein to serum protein $\geq 0.5$; (2) Ratio of pleural fluid lactate dehydrogenase (LDH) to serum $\mathrm{LDH} \geq 0.6$; and (3) Pleural fluid LDH fluid $\geq 0.67$ than the normal upper limit of serum.

The pleural fluid uric acid level was the total uric acid level in pleural fluid. Pleural fluid volume was defined as the volume of fluid in the pleural cavity that was calculated every day after removal by the pleural fluid puncture method or intrathoracic tube insertion.

The ratio of pleural fluid uric acid levels to serum was the total pleural fluid uric acid level divided by the total serum uric acid level.

Pleural fluid cholesterol level was the total cholesterol level in pleural fluid.

The ratio of pleural fluid cholesterol levels to serum was the total pleural fluid cholesterol level divided by the total serum cholesterol level.

\section{Study Instruments}

Exudates and transudates were determined using laboratory measurements of pleural fluid uric acid levels, serum uric acid levels, pleural fluid cholesterol levels, and serum cholesterol levels. The criterion for distinguishing exudates from transudates was Light's criteria.

\section{Data Analysis}

The diagnosis's accuracy was analyzed using sensitivity, specificity, and the area under the ROC (Area Under the Curve) curve with SPSS 21.

\section{Research Ethics}

This study had received approval for Dr. Moewardi Hospital's ethical eligibility with number 1.010 / VIII / HREC / 2019. 


\section{RESULTS}

\section{A.Sample Characteristics}

This study was conducted on 30 patients diagnosed with pleural effusion treated on an outpatient and inpatient pulmonology ward. Characteristics of research subjects in categorical data were presented with a frequency distribution (\%).

Characteristics of research subjects in numerical data (age, protein, LDH, glucose, cells, MN, PMN) were presented with Mean and SD. The study sample characteristics for continuous and categorical variables were shown in Table 1 and Table 2.

Table 1 showed that the 30 study subjects had an average age (Mean $=57.17 ; \mathrm{SD}=$ 13.11) years, with the youngest age being 22 years and the oldest 80 years. The average protein of pleural fluid was Mean $=3.47$;
$\mathrm{SD}=1.80$ with a minimum value of $0.50 \mathrm{ml}$ and a maximum value of $6.90 \mathrm{ml}$.

The mean serum protein (Mean= $6.50 ; \mathrm{SD}=6.65$ ) with a minimum value of 4 $\mathrm{ml}$ and a maximum value of $7.70 \mathrm{ml}$. The average pleural fluid LDH (Mean= 2025.83; $\mathrm{SD}=3466.83$ ) with a minimum value of $91 \mathrm{ml}$ and a maximum value of $15048 \mathrm{ml}$. The mean serum LDH (Mean= 579.93; $\mathrm{SD}=246.39)$ with a minimum value of $316 \mathrm{ml}$ and a maximum value of $1468 \mathrm{ml}$.

Average glucose (Mean $=77.90 ; \mathrm{SD}=$ 56.76) with a minimum value of $2 \mathrm{mg} / \mathrm{dL}$ and a maximum value of $235 \mathrm{mg} / \mathrm{dL}$. The average cell $(\mathrm{Mean}=1727.46 ; \mathrm{SD}=2822.84)$ with a minimum value of $2 / \mathrm{ul}$ and a maximum value of $14373 / \mathrm{ul}$. MN mean (Mean = 62.63; $\mathrm{SD}=37.19$ ) with a minimum value of $0 \%$ and a maximum value of $97 \%$.

Table 1. Sample characteristics (continuous data)

\begin{tabular}{lccccc}
\hline \multicolumn{1}{c}{ Variables } & n & Mean & SD & Min. & Max. \\
\hline $\begin{array}{l}\text { Age (years) } \\
\text { Protein }\end{array}$ & 30 & 57.17 & 13.11 & 22.00 & 80.00 \\
Pleura fluid (mL) & 30 & 3.47 & 1.80 & 0.50 & 6.90 \\
Serum (mL) & 30 & 6.50 & 6.65 & 4 & 7.70 \\
LDH & & & & & \\
Pleura fluid (mL) & 30 & 2025.83 & 3466.83 & 91 & 15048 \\
Serum (mL) & 30 & 579.93 & 246.39 & 316 & 1468 \\
Glukosa (mg/dL) & 30 & 77.90 & 56.76 & 2 & 235 \\
Sel $(/ \mu L)$ & 30 & 1727.46 & 2822.84 & 2 & 14373 \\
MN (\%) & 30 & 62.63 & 37.19 & 3 & 100 \\
PMN (\%) & 30 & 37.27 & 37.27 & 0 & 97 \\
\hline
\end{tabular}

\section{B. The result of bivariate analysis}

Table 2 showed female subjects were 20 patients (66.70\%). Most of the diagnoses were malignancy in 18 patients (60\%).

Comparison of sensitivity, specificity, PPV, NPV, and Likelihood Ratio (LR) against Light's criteria with transudate results

Examination of pleural fluid cholesterol, serum cholesterol, pleural fluid uric acid levels, and serum uric acid levels was shown in Table 3. Table 3 showed pleural fluid cholesterol levels had an AUC value of o.82. It meant that $82 \%$ of pleural fluid cholesterol examinations could predict light criteria results with a cut-off $<32.00$ for transudate results. The sensitivity value was o.86. It meant that $86 \%$ of the transudate diagnosis could be detected by examining pleural fluid cholesterol $<32.00$. The specificity value of pleural fluid cholesterol was o.83. It meant that $83 \%$ of patients with pleural fluid cholesterol $\geq 32.00$ were likely to exclude exudate results.

The positive predictive value (PPV) was o.6o. It meant if the pleural fluid chol- 
Sutanto et al./ Accuracy of Uric Acid and Cholesterol Levels Examination

esterol level was $<32.00$, then there was a $60 \%$ chance of a transudate result. The negative predictive value (NPV) was 0.95. It meant if the pleural fluid cholesterol level was $\geq 32$.00, then there was a 95\% chance of patients showing exudate results. The positive likelihood ratio $(\mathrm{LR}+)$ value was 4.93. It meant that the possibility of patients with pleural fluid cholesterol levels $<32.00$ will show a transudate diagnosis result of 4.93 times compared with patients with pleural fluid cholesterol levels $\geq 32$.oo.
The serum cholesterol level had an area under the curve (AUC) of 0.67. It meant that $67 \%$ of serum cholesterol tests could predict light criteria results with a cut-off of 175.50 for transudate results. The sensitivity value was 0.71 . It meant if the serum cholesterol was $\geq 175 \cdot 50$, then there was a $71 \%$ chance of patients showing transudate results. The specificity value of serum cholesterol was 0.61 . It meant that $61 \%$ of patients with serum cholesterol $<175.50$ were likely to exclude exudate results.

Table 2. Sample characteristics (categorical data)

\begin{tabular}{llcc}
\hline \multicolumn{1}{c}{ Characteristics } & \multicolumn{1}{c}{ Categories } & $\begin{array}{c}\text { Frequency } \\
\text { (n) }\end{array}$ & $\begin{array}{c}\text { Percentage } \\
\text { (\%) }\end{array}$ \\
\hline \multirow{2}{*}{ Gender } & Male & 10 & 33.30 \\
& Female & 20 & 66.70 \\
Diagnosis & Infection & 7 & 23.30 \\
& Malignancy & 18 & 60 \\
& Others & 5 & 16.70 \\
\hline
\end{tabular}

Table 3. Sensitivity, specificity, PPV, NPV, and LR of research variables against Light's Criteria with transudate results

\begin{tabular}{lcccccccc}
\hline \multicolumn{1}{c}{ Variables } & Cut-off & Sensitivity & Specificity & AUC & PPV & NPV & LR & p \\
\hline $\begin{array}{l}\text { Pleural fluid } \\
\text { cholesterol }\end{array}$ & $<32.00$ & 0.86 & 0.83 & 0.82 & 0.60 & 0.95 & 4.93 & 0.012 \\
$\begin{array}{l}\text { Serum } \\
\text { cholesterol }\end{array}$ & $\geq 175.50$ & 0.71 & 0.61 & 0.67 & 0.36 & 0.88 & 1.83 & 0.194 \\
$\begin{array}{l}\text { Pleural fluid } \\
\text { uric acid levels }\end{array}$ & $\geq 7.25$ & 0.86 & 0.87 & 0.83 & 0.67 & 0.95 & 6.57 & 0.009 \\
Serum uric acid & $\geq 7.10$ & 0.86 & 0.70 & 0.65 & 0.46 & 0.94 & 2.82 & 0.249 \\
\hline
\end{tabular}

The positive predictive value (PPV) was 0.36. It meant that if the serum cholesterol level was $\geq 175 \cdot 50$, then there was a $36 \%$ chance of a transudate result. The negative predictive value (NPV) was o.88. It meant that if the serum cholesterol level was $<175.50$, then there was an $88 \%$ chance of an exudate result. The positive likelihood ratio ( $\mathrm{LR}+$ ) was 1.83 . It meant that the likelihood of a patient with a serum cholesterol level of 175.50 would get a transudate diagnosis was 1.83 times compared to a patient with a serum cholesterol level $<175.50$.

Pleural fluid uric acid levels showed the highest value of the under-curve area
(AUC), which was o.83. It meant that $83 \%$ of pleural fluid uric acid examinations could predict the results of light criteria with a cut-off of 7.25 for transudate results. The sensitivity value was 0.86 . It meant that if the uric acid pleural fluid was $\geq 7.25$, then there was an $86 \%$ chance of the transudate result. The specificity value of pleural fluid uric acid was 0.87. It meant that if the pleural fluid uric acid was $<7.25$, then there was an $87 \%$ chance of exudate results.

In this study, it was obtained a positive predictive value (PPV) of 0.67 . It meant that if the uric acid levels in the pleural 
fluid were $\geq 7.25$, then there was a $67 \%$ chance of a transudate result. Meanwhile, the negative predictive value (NPV) was 0.95. It meant that if the pleural fluid uric acid levels were $<7.25$, then there was a 95\% chance of patients with exudate results. The positive likelihood ratio $(\mathrm{LR}+)$ was 6.57. It meant that patients with pleural fluid uric acid levels $\geq 7.25$ will get a transudate diagnosis of 6.57 times compared with patients compared to uric acid levels $<7.25$.

The serum uric acid level had an AUC value of 0.65 . It meant that $65 \%$ of serum uric acid tests could predict the results of Light's criteria with a cut-off of 7.10 for transudate results. The sensitivity value was o.8. It meant that if the serum uric acid $\geq 7.10$, then there were $86 \%$ of the transudate results. The specificity value of serum uric acid obtained in this study was 0.70. It meant that $70 \%$ of patients with serum uric acid $<7.10$ were likely to be excluded.

The positive predictive value (PPV) was 0.46 . It meant that if the serum uric acid level was $\geq 7.10$, then there was a $46 \%$ chance of a transudate result. The negative predictive value (NPV) was 0.94. It meant that if the serum uric acid level was $<7.10$, then there was a 94\% chance of patients with exudate results. The positive likelihood ratio $(\mathrm{LR}+)$ was 2.82 . It meant that the likelihood of a patient with a serum uric acid level $\geq 7.10$ will get a transudate diagnosis was 2.82 times compared to a patient who had a serum uric acid level <7.10.

Pleural fluid cholesterol levels $(\mathrm{p}=$ 0.012) and pleural fluid uric acid ( $p=$ o.009) can be suggested as predictors of Light's criteria results because the ROC curve results obtained statistically significant results. The variables that were not recommended as predictors of Light criteria were serum cholesterol $(p=0.194)$ and serum uric acid ( $p=0.249)$ because they were not statistically significant.

\section{DISCUSSION}

The total subjects of this study were 30 patients. Based on Light's criteria, 23 patients had exudates and 7 patients' transudates. The most pleural effusion patients were women, 20 (66.70\%) with 15 exudates (65.20\%) and 5 transudates (71.40\%). Jain et al. (2015) reported that the largest gender was male, 38 patients (63\%), with 16 exudates (53\%) and 22 transudates (73\%). The ratio of male and female pleural effusions was 1.3: 1.

Distribution of pleural effusions by sex with a TB diagnosis was the main cause of effusion in men, while malignancy was the main cause in women. As many as $61.70 \%$ of patients with malignancy was $\geq 50$ years and $76.60 \%$ of TB effusion patients aged <40 years (Light, 2013). These results were consistent with this study's characteristics, where the mean age was 57.13 years ( $\mathrm{SD}=13.11)$, and the most common disease was malignancy as many as 18 cases (60\%).

This study found that the sensitivity and specificity values of pleural fluid cholesterol levels were $86 \%$ and $83 \%$, with a cut-off value of 32.00. An AUC value of $82.00 \%$ which indicated a combination of moderate sensitivity and specificity (AUC $=$ o.82; $\mathrm{p}=0.012$ ).

The sensitivity and specificity values of examining serum cholesterol levels were $71 \%$ and $61 \%$, respectively, with a cut-off value of 175.50 . The receiver operating characteristics (ROC) curve showed an AUC value of $0.67(\mathrm{p}=0.194)$.

Hamal et al. (2013) reported that in transudate pleural effusions, cholesterol levels in the pleural fluid were Mean=0.53; $\mathrm{SD}=0.28 \mathrm{mmol} / \mathrm{L}$. Pleural fluid cholesterol levels had a sensitivity of $97.7 \%$ and a speci- 
ficity of $100 \%$ for the diagnosis of exudates with PPV 100\%.

It was found that this criterion incorrectly classified only one case of malignant effusion as a transudate. That misclassification also occurred in the use of Light's criteria. Misclassified exudate showed a low concentration of cell components because the pleura had just been affected by a tumor (Hamal et al., 2013).

Patel and Choudhury's (2013) study found that the transudate's average cholesterol level was $30 \mathrm{mg} / \mathrm{dL}$. By using a 60 $\mathrm{mg} / \mathrm{dL}$ cut-off to separate exudate from transudate, only $5 \%$ were misclassified. Pleural fluid cholesterol levels found at cutoff $\geq 60 \mathrm{mg} / \mathrm{dL}$ and/ or total protein at cutoff $\geq 3 \mathrm{~g} / \mathrm{dL}$ was used to differentiate transudates and exudates, sensitivity, specificity, positive predictive value (PPV), and negative predictive value (NPV) were 100\% (Patel and Choudhury, 2013).

Other findings showed pleural cholesterol levels $\geq 55 \mathrm{mg} / \mathrm{dL}$ and pleural cholesterol/ serum $\geq 0.3$ to determine exudative effusion with 93\% sensitivity, 100\% specificity, 100\% PPV, and 95.2\% accuracy (Hamal, 2018).

Pleural cholesterol was thought to originate from degenerative cells and vascular leakage from increased permeability. Although the cause of the elevated cholesterol level in pleural exudate was unknown, two possibilities had been described. First, cholesterol was synthesized by pleural cells for their own needs. Second, the concentration of cholesterol in the pleural cavity increases due to degeneration of the leukocytes and erythrocytes. Increased pleural capillary permeability in pleural effusion patients will allow plasma cholesterol to enter the pleural cavity (Hamal et al., 2013).

Uric acid is a weak acid found in extracellular plasma fluid, especially in the form of monosodium urate. Uric acid is known as the end product of purine metabolism. At physiological body $\mathrm{pH}$ ( $\mathrm{pH}$ 7.4), uric acid is easily filtered from plasma. Protein-bound uric acid is present in very small amounts. Therefore, uric acid is freely filtered in the blood circulation. Increased uric acid can be found in clinical conditions associated with tissue hypoxia (Oliveira and Burini, 2012).

In this study, the sensitivity and specificity values of pleural fluid uric acid levels were $86 \%$ and $87 \%$, respectively, at a cut-off value of 7.25 to differentiate exudates and transudates in pleural effusion disease. With an AUC value of $83.20 \%(\mathrm{p}=$ o.009), cut-off $=7.25$ provided optimal sensitivity and specificity.

The study of Uzun et al. (2000) reported that serum and pleural uric acid levels were higher in transudates than in exudates ( $p<0.001)$. The specificity and sensitivity of pleural uric acid for the diagnosis of transudate effusions were $73 \%$ and $80.6 \%$, respectively. The specificity and sensitivity of pleural uric acid for the diagnosis of transudate effusion from exudate without malignancy were $71.8 \%$ and $91.7 \%$, respectively.

Research by Jain et al. (2015) reported that the cut-off value of pleural fluid uric acid to differentiate transudates from exudates was $5.5 \mathrm{mg} / \mathrm{dL}$ with a sensitivity of $94 \%$ and a specificity of $83 \%$.

Another study showed the pleural fluid uric acid cut-off value to differentiate transudates from exudates was $5.35 \mathrm{mg} /$ $\mathrm{dL}$, with a sensitivity of $89.32 \%$ and a specificity of 92.6\% (Hazarika et al., 2015). Increased pleural fluid uric acid levels occur in transudate pleural effusions. Increased permeability, due to changes in pleuralcapillary pressure in transudate formation, is the cause of increased uric acid levels in pleural fluid (Uzun, 2000). 
This study found the sensitivity and specificity of examining serum uric acid levels of $86 \%$ and $70 \%$, respectively, at a cut-off value of 7.10 to differentiate exudates and transudates in pleural effusion disease. Based on the ROC curve, the AUC value was 0.65 with a $\mathrm{p}=0.249$.

Kumar et al. (2020) found that mean serum uric acid levels and pleural fluid uric acid levels were higher in subjects with transudate type pleural effusions when compared to subjects with exudate type, and the differences were found to be statistically significant $(\mathrm{p}=0.009, \mathrm{p}=0.033)$. These results reflected the utility of examining uric acid levels in differentiating transudates from exudates. Low uric acid levels shown in exudative pleural effusions were secondary to local factors that contributed to increase or decrease capillary permeability or obstruction of lymphatic flow.

Whereas in transudate-type pleural effusions, there was an imbalance in hydrostatic and oncotic forces without changes in capillary permeability (Pergulwar et al., 2016). Increased uric acid in pleural fluid can be caused by tissue hypoxia, in which uric acid synthesis was regulated in tissue hypoxia and oxidative stress (Kumar et al., 2020).

The inadequacy of the ratio of pleural fluid uric acid levels to serum can be attributed to the limitations of this study because the use of previous treatments that can affect uric acid levels such as uricosuric drugs, diuretics, and chemotherapy was not controlled (Antony, 2003; Putra and Yunus, 2013; Light, 2013).

Uricosuric drugs used in chronic kidney reduce uric acid levels (Light, 2013; Mason et al., 2010). Diuretic therapy in pleural effusion patients can increase uric acid levels. Diuresis causes water to leave the pleural space faster than protein and LDH (Light, 2013; Grippi et al., 2015).

Chemotherapy can increase uric acid levels because the degradation of dead tumor cells will be converted into uric acid (Jain et al., 2015; Grippi et al., 2015). The examination of pleural fluid cholesterol and uric acid levels had sufficiently high sensitivity and specificity to distinguish exudates and transudates in pleural effusions. Both of these tests can be considered routine examinations in pleural effusion cases, which require determining the type of effusion.

\section{AUTHOR CONTRIBUTION}

Yusup Subagyo Sutanto contributed to the concept, design, analysis, and discussion of data.

\section{CONFLICT OF INTEREST}

This study was self-funded.

\section{FUNDING AND SPONSORSHIP}

There was no conflict of interest.

\section{ACKNOWLEDGEMENT}

Our thanks went to the Department of Pulmonology and Respiratory Medicine, Faculty of Medicine, Sebelas Maret University, for their support in this research.

\section{REFERENCE}

Antony VB (2003). Immunological mechanisms in pleural disease. Eur Respir J. 21(3): 539-544. doi: 10.1183/-09031936.-03.00403902.

Bansal A, Tandon S, Kharb S (2010). Diagnostic value of uric acid in pleural effusion. Zhongguo Fei Ai Za Zhi. 13(4): 349-351. https://doi.org/10.3779/j.issn.1009-3419.2010.04.15.

Mason RJ, Broaddus VC, Martin TR, King TE, Schraufnagel DE, Murray JE, Nadel JA (2010). Murray and Nadel's 
Textbook of Respiratory Medicine. Fifth Edition. Philadelphia: Elsevier Saunders, 1719-63.

Bouros D (2009). Pleural Disease. Second Edition. New York: Marcel Dekker. 237-52.

Grippi MA, Elias JA, Fishman JA, Kotloff RM, Pack AI, Senior RM (2015). Fishman's Pulmonary Diseases and Disorders. Fifth Edition. New York: McGraw-Hill Education, 1168-1187.

Jain A, Jain R, Petkar SB, Gupta SK, Khare N, Dutta J (2015). A study of uric acid - a new biochemical marker for the differentiation between exudates and transudates in a pleural effusion cases. National Journal of Community Medicine. 5(2): 204-208.

Light RW (2013). Pleural Diseases. Sixth Edition. Philadelphia: Lippincott Williams \& Wilkins, 128-39.

Maskell NA, Butland RJA (2003). BTS guidelines for the investigation of a unilateral pleural effusion in adults. Thorax. 58: 8-17.
Oliveira EP, Burini RC (2012). High plasma uric acid concentration: causes and consequences. BioMed Central. 4(12): 1-7. doi: 10.1186/1758-5996-4-12.

Peng M-J, Wang N-S (2004). Anatomy of the pleura. In: Bouros D.(ed.). Pleural Disease. New York: Marcel Dekker, Inc, pp.23-40.

Porcel JM (2013). Identifying transudates misclassified by Light's criteria. Curr Opin Pulm Med. 19(4): 362-367. doi: 10.1097/MCP.obo13e32836022-dc.

Putra I, Yunus F (2013). Pleural Anatomy and Physiology. 40(6): 407-412.

Richard WL (2013). The Light criteria: the beginning and why they are useful 40 years later. Clin Chest Med. 34(1): 2126. doi: 10.1016/j.ccm.2012.11.006.

Yalcin NG, Choong CKC, Eizenberg N (2013). Anatomy and pathophysiology of the pleura and pleural space. Thorac Surg Clin. 23(1): 1-10. doi: 10.1016/j.thorsurg.2012.10.008. 